\title{
Метод сейсмоизоляции и виброизоляции, основанный на свойствах изображений Фурье финитных функций
}

\author{
Е.Н.Курбацкий, РУТ (МИИТ), Москва \\ Е.Ю.Титов, РУТ (МИИТ), Москва \\ О.А.Голосова, РУТ (МИИТ), Москва \\ С.С.Харитонов, РУТ (МИИТ), Москва
}

Предложен метод сейсмоизоляции и виброизоляции, основанный на свойствах изображений Фурье финитных функций. Разработаны модели для определения динамических свойств гранулированных материалов из бытовых полимерных отходов и из изношенных шин. В будущем полимерные бытовые материалы могут стать неиссякаемым источником для получения материала, обладающего сейсмоизолирующими и виброизолирующими свойствами. Разработка способов использования полимерных бытовых отходов для сейсмоизоляции и виброизоляции позволит одновременно решать две проблемы: защищать от загрязнений окружающую среду и обеспечивать сейсмостойкость сооружений.

Ключевые слова: преобразование Фурье, финитные функции, целые функции, полимерный материал, скорости волн, сейсмоизоляция, виброизоляция.

Seismic Insulation and Vibroinsulation Method, Based on the Properties of Fourier Images of Finite Functions Курбацкий Е.Н., РУТ (МИИТ), Москва

Титов Е.Ю., РУТ (МИИТ), Москва

Голосова 0.А., РУТ (МИИТ), Москва

Харитонов С.С., РУТ (МИИТ), Москва

A method of seismic isolation and vibration isolation based on the properties of Fourier transforms of finite functions is proposed. Models have been developed to determine the dynamic properties of granular materials from household polymer waste from worn tires. In the future, polymeric household materials may become an inexhaustible source for obtaining material with seismic and vibration isolating properties. The development of methods for the use of polymeric household waste for seismic isolation and vibration isolation will solve two problems facing the world's population: protect against environmental pollution and ensure earthquake resistance of structures.

Keywords: fourier transform, finite functions, entire functions, polymer material, wave velocities, seismic isolation, vibration isolation

\section{Введение}

Больше половины населения земного шара проживает в районах с повышенной сейсмической активностью, поэтому очень важно уметь проектировать и строить сейсмостойкие сооружения. Существует много способов снизить сейсмические воздействия до безопасных. Существует много методов и программных комплексов для проектирования сейсмостойких сооружений. В настоящей работе используется метод решения задач строительной механики и теории упругости, основанный на свойствах изображений Фурье финитных функций [1]. Считаем, что этот метод имеет некоторые преимущества при решении динамических задач по сравнению с известными методами и используется для оценки сейсмоизолирующих и виброизолирующих свойств слоёв гранулированных материалов.

Известно большое количество типов сейсмоизолирующих устройств, которые могут использоваться для защиты мостов, зданий, различного оборудования и в атомной энергетике. Многие из них весьма дорогостоящие. Мы предлагаем в качестве сейсмоизоляции и виброизоляции использовать гранулированные материалы, полученные из переработанных твёрдых бытовых отходов и отработанных шин, что значительно дешевле дорогостоящей сейсмоизоляции и виброизоляции. Кроме того, попутно решается проблема утилизации бытовых отходов и изношенных шин.

В последние годы утилизация твёрдых коммунальных отходов (ТКО) стала значительной экологическая проблемой. В России ежегодно образуется 55-60 млн тонн отходов. Образование твёрдых коммунальных отходов в Московском регионе составляет порядка 4,7 млн тонн ежегодно. Прирост образования ТКО в Московском регионе по данным Гринпис (Greenpeace) - 3\% в год, таким образом к 2025 году объём образования составит порядка 5,6 млн тонн в год .

Такую же серьёзную проблему представляет собой утилизация изношенных автомобильных шин. 800 миллионов утильных шин появляется каждый год во всём мире как следствие огромного роста количества автомобилей. Только в США ежегодно появляется около 300 млн утильных шин и ожидается, что это количество ежегодно будет расти примерно на $2 \%$ [15].

Переработка бытовых полимерных отходов с целью превращения мусора в гранулированный материал позволяет повторно использовать этот материал для виброизоляции и сейсмоизоляции, для чего необходимо определить динамические свойства сыпучих материалов из пластика. С этой целью разработан метод определения динамических свойств гранулированных материалов из пластика. 
Считается, что переработанный полимерный пластик и резина в будущем станут важным необходимым материалом для защиты от землетрясений и вибраций, а бытовые отходы и шины потенциально могут стать источником этого материала. Таким образом, одновременно решаются две проблемы: защита от землетрясения и удаление отходов.

\section{1. Математические основы метода и область применения}

В основе метода решения задач упругости и строительной механики лежит теорема Пэли-Винера-Шварца [1]. Эта теорема утверждает, что изображением Фурье финитной функции является целая функция.

Функции, тождественно равные нулю вне конечного интервала $(a, b)$ (вне ограниченной области $\Omega$ ), называются финитными [2].

Целой функцией называется аналитическая функция комплексного переменного, представимая всюду сходящимся степенным рядом и, следовательно, не имеющая особенностей ни в какой ограниченной области плоскости Z. В многомерном случае $Z=(z 1, \ldots . . . z n)$ [2].

Теорема о делимости многочлена (теорема Безу): «Многочлен, целый относительно $Z$, делится на одночлен $(Z-\alpha)$ без остатка или имеет остаток, равный тому значению делимого, который оно принимает при $Z=a »[2]$.

Теорема Винера-Пэли-Шварца: «Преобразование Фурье финитной функции $f(z)$, обращающейся в нуль при $|z| \geq a$, если целая аналитическая функция $\varphi$ переменного $s=\sigma+i \tau$, при каждом $q=0,1,2 \ldots$ удовлетворяющая неравенству $\left|s^{q} \varphi(s)\right|$ $\leq c_{q} e^{\alpha|\tau| q} \gg$. Справедливо и обратное утверждение.

\section{2. Теорема о граничных функциях}

Теорема Винера-Пэли-Шварца позволяет доказать теорему, устанавливающую связь между значениями функции на границе области в краевой задаче и значениями изображений Фурье правой части уравнений.

Пусть $\Omega$ - ограниченная область с границей $S$ и

$\theta(\Omega)=\left\{\begin{array}{l}1, x \in \Omega \\ 0, x \notin \Omega\end{array}\right.$ - характеристическая функция этой области.

Тогда $U(x)=\{U(x)\} \theta(\Omega)$ - финитная функция.

Пусть $\varsigma$ - дифференциальный оператор с постоянными коэффициентами в области $\Omega$. Применяя оператор $\varsigma$ к функции $U(x)$, получим дифференциальное уравнение, записанное в обобщённых функциях:

$$
\varsigma U=q(x)+\sum \mu_{\mathrm{k}} \delta^{(k)}(S)+\sum \gamma_{\mathrm{k}} \delta^{(k)}(S)
$$

где $q(x)$ - в задачах механики это внешняя нагрузка; $\delta^{(k)}(S)$ - дельта-функции и их производные, сосредоточенные на границе области $S ; \mu_{\mathrm{k}}$ - заданные значения функции $U(x)$ и её производных по нормали к границе $S ; \gamma_{\mathrm{k}}$ - неизвестные значения функции $U(x)$ и её производных по нормали к границе $S$;

Теорема: «Значения неизвестных функций и их производных на границе области $S$ определяются значениями изобра- жений Фурье правой части уравнения (нагрузки) на нулевых множествах полинома, соответствующего оператору ऽ» [1; 3].

Доказательство: применим преобразование Фурье к дифференциальному уравнению (1) по всем переменным и найдем изображение Фурье функции $U(x)$ :

$$
\tilde{U}(v)=\frac{\tilde{q}(v)+F\left(\sum \mu_{k} \delta^{(k)}(S)\right)+F\left(\sum \gamma_{k} \delta^{(k)}(S)\right)}{\varsigma(v)},
$$

где $v$ - параметр преобразования Фурье, $\tilde{q}(v), F\left(\sum \mu_{\mathrm{k}} \delta^{(k)}(S)\right)$ и $F\left(\sum \gamma_{k} \delta^{(k)}(S)\right)$ - изображения Фурье функций $q(x), \Sigma \mu k \delta(k)$ (S) и $\sum \mu_{\mathrm{k}} \delta^{(k)}(S)$.

Так как $U(x)$ - функция финитная, то изображение Фурье этой функции - $\tilde{U}(v)$, должно быть целой функцией. С другой стороны, знаменатель выражения (2) представляет собой полином не нулевой степени и, следовательно, превращается в нуль на некоторых множествах в соответствии с основной теоремой алгебры (в одномерном случае эти множества состоят из изолированных точек).

Числитель выражения (2) представляет собой целую функцию, так как равен сумме изображений Фурье финитных функций. Поэтому для того, чтобы функция $U(v)$ была целой, необходимо и достаточно, чтобы нулевые множества числителя содержали нулевые множители знаменателя, то есть числитель должен превращаться в нуль на нулевых множествах знаменателя:

$\tilde{q}(v)+F\left(\sum \mu_{\mathrm{k}} \delta^{(k)}(S)\right)+F\left(\sum \gamma_{\mathrm{k}} \delta^{(k)}(S)\right)=0, \forall v \in C^{n}: \varsigma(v)=0$.

Что и требовалось доказать. В этом случае числитель будет делиться на знаменатель без остатка.

\section{3. Построение граничных элементов}

Рассмотрим ограниченную область $\Omega$, упругой среды, в течение интервала времени $(0, T)$ под действием нагрузки $f_{j}$. Представим смещения и напряжения в декартовой системе координат $\left(x_{1}, x_{2}, x_{3}\right)$ в виде финитных функций:

$U_{\mathrm{i}}(x, t)=\left\{U_{\mathrm{i}}\left(x_{1}, x_{2}, x_{3}\right)\right\} \theta(\Omega) \theta(T) ; \sigma_{i j}=\left\{\sigma_{i j}\right\} \theta(\Omega) \theta(T) ;$ где $\theta(\Omega)$ и $\theta(\mathrm{T})$ - характеристические функции области $\Omega$ во временном интервале $(0, T)$.

Представим, дифференциальные уравнения теории упругости для ограниченной области в обобщённых функциях: $\mu U_{j, i}+(\lambda+\mu) U_{i, j j}-\rho \ddot{U}_{j}=-F_{j}+\left[\sigma_{i j}\right]_{s} \cos \left(\bar{n} \cdot x_{i}\right) \delta_{s}+\lambda\left[\left[U_{j}\right]_{s} \cos \left(\pi \cdot x_{i}\right) \delta_{s}\right]_{j}+$ $+\mu\left[\left[U_{j}\right]_{s} \cos \left(\bar{n} \cdot x_{j}\right) \delta_{s}\right]_{j,}+\mu\left[\left[U_{j}\right]_{s} \cos \left(\bar{n} \cdot x_{i}\right) \delta_{s}\right]_{, j}-\rho\left[U_{j}\right]_{t=0} \dot{\delta}(t)+$ $\rho\left[U_{j}\right]_{t=T} \dot{\delta}(t-T)-\rho\left[\dot{U}_{j}\right]_{t=0} \delta(t)+\rho\left[\dot{U}_{j}\right]_{t=T} \delta(t-T)$.

Здесь функции $\left[U_{i}\right]_{s}$ и $\left[\sigma_{\mathrm{i}, \mathrm{j}}\right]_{s}$ представляют собой скачки функций $U_{i}$ и $\sigma_{\mathrm{i}, \mathrm{j}}$ при пересечении границ $S$ по нормали из области $\Omega$. Так как эти функции вне области равны нулю, они представляют собой граничные функции.

$\left[U_{j}\right]_{t=0} ;\left[U_{j}\right]_{t=T^{\prime}}\left[\dot{U}_{j}\right]_{t=0}$ и $\left[\dot{U}_{j}\right]_{t=T}-$ функции, определяющие начальные и конечные условия.

Функции $F_{j}$ совпадают с функциями $f_{j}$ внутри области $\Omega$ в течение интервала времени $(0, T)$ и равны нулю вне этой области и вне этого интервала. Правая часть уравнения (4) включает в себя функции, описывающие взаимодействие фи- 
нитной области с окружающим массивом, то есть функции напряжений, деформаций и перемещений на границах области.

Применив преобразование Фурье к уравнениям (4), получим:

$$
\left[v_{1}^{2}+v_{2}^{2}+v_{3}^{2}-\eta^{2} \omega^{2}\right] \widetilde{U}_{i}+\left(\eta^{2}-1\right) \widetilde{U}_{j} v_{i} v_{j}=\tilde{X}_{i} / \mu
$$

Здесь $v_{1}, v_{2}, v_{3}$ - параметры преобразования Фурье, соответствующие пространственным координатам;

$\omega$ - параметр преобразования, соответствующий координате $\tau(\tau=\alpha t)$;

$\eta=\frac{\alpha}{\beta}$, где $\alpha$ и $\beta$ скорости распространения продольных и поперечных волн;

$\tilde{U}_{i}$ - преобразование Фурье функции $U_{j}$ (перемещения);

$X_{i}$ - преобразование Фурье правой части уравнения (4).

Все функции уравнения (5) целые, поскольку они являются преобразованиями Фурье финитных функций.

Для решения динамических задач теории упругости был разработан плоский прямоугольный граничный элемент (рис. 1).

Опишем кратко построение зависимостей между функциями на гранях элемента. Представим уравнения теории упругости для плоского прямоугольного элемента в обобщённых финитных функциях. В правых частях этих уравнений появятся функции, описывающие напряжения и перемещения граней (всего 16 функций).

Для каждой стороны элемента необходимо определить четыре граничных функции $U, V, \sigma, \tau$ (соответствующие горизонтальным и вертикальным перемещениям, нормальным и тангенциальным напряжениям). Начальные конечные условия отсутствуют, так как рассматриваются гармонические колебания.

Применим преобразование по двум пространственным координатам и по времени.

Выполнив необходимые преобразования, можно получить два уравнения для изображений Фурье функций перемещений.

Для определения 16-ти граничных функций необходимо иметь 16 условий. Восемь из них могут быть получены из условий на границах каждого элемента или из условий равенства перемещений и напряжений на сторонах соседнего элемента. Оставшиеся восемь уравнений могут быть получены на основании теоремы о граничных функциях. Эти восемь условий можно записать в виде восьми алгебраических уравнений.

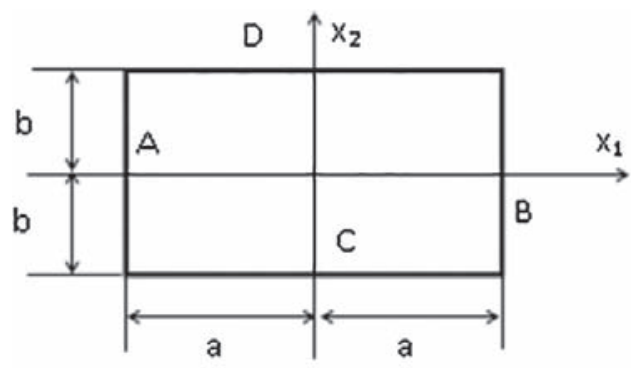

Pис. 1. Плоский прямоугольный элемент
Предполагая, что размеры элементов малы по сравнению с длиной волны, уравнения могут быть упрощены, и после ряда преобразований представлены в виде:

$$
\begin{aligned}
& \text { 1) }\left[\widetilde{U}_{C}+\widetilde{U}_{D}\right]-\left[\widetilde{U}_{A}+\widetilde{U}_{B}\right]=0 \\
& \text { 2) } \frac{\left[\tilde{\tau}_{C}+\tilde{\tau}_{D}\right]}{2}+\mu\left(\frac{\left[\widetilde{U}_{C}-\widetilde{U}_{D}\right]}{2 b}+\frac{\left[\widetilde{V}_{A}-\widetilde{V}_{B}\right]}{2 a}\right)=0 \\
& \text { 3) } \frac{4 a b \rho \omega^{2}\left[\widetilde{U}_{A}+\widetilde{U}_{B}\right]}{2}-2 b\left[\widetilde{\sigma}_{A}-\widetilde{\sigma}_{B}\right]-2 a\left[\tilde{\tau}_{C}-\tilde{\tau}_{D}\right]=0 \\
& \text { 4) } \frac{\left[\widetilde{\sigma}_{A}+\widetilde{\sigma}_{B}\right]}{2}+\frac{(\lambda+2 \mu)\left[\widetilde{U}_{A}-\widetilde{U}_{B}\right]}{2 a}+\frac{\lambda\left[\widetilde{V}_{C}-\widetilde{V}_{D}\right]}{2 b}=0 \\
& \text { 5) } \frac{4 a b \rho \omega^{2}\left[\tilde{V}_{C}+\widetilde{V}_{D}\right]}{2}-2 a\left[\widetilde{\sigma}_{C}-\widetilde{\sigma}_{D}\right]-2 b\left[\tilde{\tau}_{A}-\tilde{\tau}_{B}\right]=0 \\
& \text { 6) } \frac{\left[\widetilde{\sigma}_{C}+\widetilde{\sigma}_{D}\right]}{2}-\frac{\lambda\left[\widetilde{U}_{A}-\widetilde{U}_{B}\right]}{2 a}+\frac{(\lambda+2 \mu)\left[\widetilde{V}_{C}-\widetilde{V}_{D}\right]}{2 b}=0 \\
& \text { 7) } \frac{\left[\widetilde{V}_{A}+\widetilde{V}_{B}\right]}{2}-\frac{\left[\widetilde{V}_{C}+\widetilde{V}_{D}\right]}{2}=0 \\
& \text { 8) } \frac{\left[\tilde{t}_{A}+\tilde{\tau}_{B}\right]}{2}-\frac{\left[\tilde{\tau}_{C}+\tilde{\tau}_{D}\right]}{2}=0 \text {. }
\end{aligned}
$$

Все входящие в систему уравнений неизвестные представляют собой изображения Фурье по соответствующей пространственной координате при параметре преобразования Фурье, равном нулю, то есть представляют собой интегралы от функций. Например:

$$
\tilde{U}_{c}(v)=\int_{-a}^{a} u_{c}(x) e^{i v x} d x \text { nри } v=0 \quad \tilde{U}_{c}(0)=\int_{-a}^{a} u_{c}(x) d x
$$

Восемь уравнений, приведённых в системе (6), имеют ясный физический смысл:

- уравнения 3 и 5 соответствуют уравнениям движения центров масс;

- уравнения 2, 4 и 6 соответствуют закону Гука;

- уравнение 8 соответствует равенству касательных напряжений;

- уравнения 1 и 7 соответствуют закону непрерывности.

\section{4. Динамические модели гранулированных сред}

Искусственные материалы, используемые для сейсмо- и виброзащиты, могут быть гранулированными полимерами, смесями гранулированных полимеров с песком или специально разработанным материалом с заданными свойствами. Для получения гранулированного материала можно также использовать изношенные шины.

Имеется возможность разработать сыпучий материал с необходимыми характеристиками. Определим некоторые свойства гранулированных материалов, которые могут быть полезны для сейсмоизоляции. А именно: рассмотрим особенности распространения волн напряжений в природных и искусственных гранулированных средах [4].

В последнее время усилия многих исследователей были направлены на создание моделей гранулированных сред, состоящих из частиц различных форм и размеров, упакованных случайным образом. На кафедре «Подземные сооружения» МИИТа исследовались динамические свойства искусственной среды, состоящей из смеси гранул 
полимерного материала (полиэтилена) и песка. Размеры гранул полимерного материала значительно больше диаметров песчинок. Целью исследований являлась разработка сыпучей среды, обладающей определёнными свойствами, для использования её в качестве сейсмоизолирующих и виброизолирующих слоёв.

Рассмотрим модели, описывающие распространение продольных и поперечных волн в гранулированных средах: в природной среде (песок) и искусственной (гранулы из полимерного материала с песком). Возможны различные виды упаковок сфер. Этот вопрос интересовал многих учёных. В частности, известен спор Ньютона с шотландским астрономом Джеймсом Грегори. В настоящей работе будем рассматривать простую упаковку. Схема расположения сфер в кубическом элементе пористых сред представлена на рисунке 2.

При рассмотрении искусственных сред будем считать, что песчинки заполняют пустоты между гранулами полиэтилена. Рассмотрим среду, состоящую из сфер одинаковых размеров и одинакового материала.

Простая модель, которая привлекла внимание многих исследователей, представляет собой среду, состоящую из упругих сфер, сжатых некоторой системой начальных напряжений и подвергнутых дополнительным добавочным напряжениям, которые возникают при прохождении упругих волн. Динамическое поведение сред полностью описывается скоростями распространения продольных и поперечных волн и параметром, определяющим затухание.
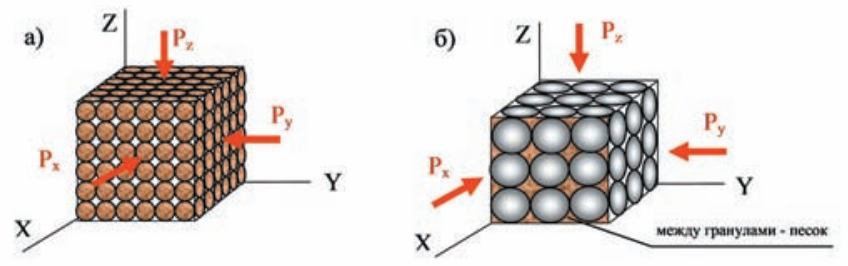

Рис. 2. Элементарные объёмы природной (а) и искусственной (б) сред
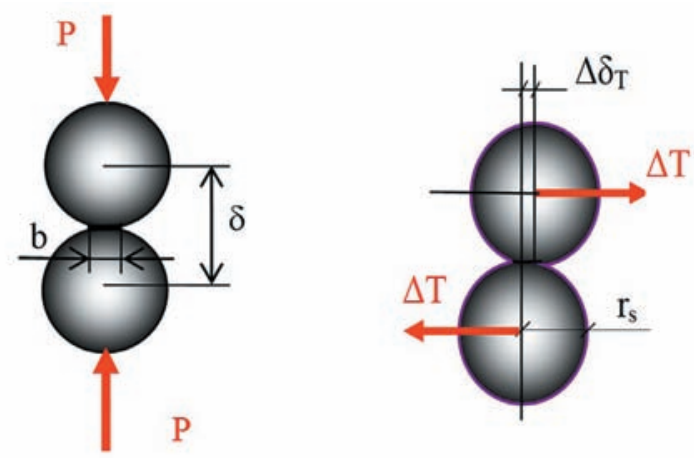

Рис. 3. Модели для определения смешений при действии нормальных напряжений и напряжений сдвига: $b$ - диаметр контактной площадки между сферами; $r_{\text {s }}$ - радиус сфер; $\Delta \delta_{T}$ - приращение относительного смещения центров двух контактирующих сфер при изменении сдвигающей силы на величину $\Delta T$
Для определения скоростей распространения упругих волн в гранулированных средах упругие константы определяются как отношения средних напряжений к средним деформациям. Для определения деформаций используется теория контактных напряжений Герца. Модели, используемые для определения средних деформаций, представлены на рисунке 3. На рисунках моделей показаны параметры, которые используются при определении деформаций. Подробный вывод выражений для определения скоростей распространения продольных и поперечных волн в гранулированных средах можно найти в работах [4-6]. Приведём краткое изложение этой теории.

Используя средние нормальные напряжения и средние деформации, можно получить средний модуль упругости для гранулированных сред. Пропуская промежуточные выкладки, получим:

$$
\tilde{E}=\frac{\tilde{p}_{x x}}{\tilde{\varepsilon}_{x x}}==\left[\frac{3 E_{s}^{2} \tilde{p}}{8\left(1-v_{s}^{2}\right)^{2}}\right]^{1 / 3} .
$$

Осреднённую плотность среды можно найти, разделив массу сферы на объём куба, в который она вписывается. Имеем:

$$
\tilde{\rho}=\frac{4 \pi r_{s}^{3}}{3 \cdot 8 r_{s}^{3}} \rho_{s}=\frac{\pi \rho_{s}}{6},
$$

где $\rho_{\text {s }}$ - плотность материала.

Скорость распространения продольных волн в гранулированной среде, сложенной из одинаковых сфер, нагруженных давлением $\tilde{p}$, вдоль оси, проходящей через точки контакта сфер, определяется выражением:

$\alpha_{p}=\left[\frac{3 E_{s}^{2} \tilde{p}}{8\left(1-v_{s}^{2}\right)^{2}}\right]^{1 / 6} \cdot\left[\frac{6}{\pi \rho_{s}}\right]^{1 / 2}=\left[\frac{3^{4} E_{s}^{2} \tilde{p}}{\pi^{3} \rho_{s}^{3}\left(1-v_{s}^{2}\right)^{2}}\right]^{1 / 6}$.

Как следует из анализа последнего выражения, скорости распространения продольных волн возрастают пропорционально корню шестой степени давления в грунтовом массиве, что достаточно хорошо соответствует экспериментам, выполненным в полевых условиях.

Для определения модуля сдвига необходимо определить отношение средних напряжений к средним деформациям сдвига. Для этой цели, следуя работе Миндлина [5], рассмотрим элемент гранулированной среды, подверженной деформации, аналогичной деформации простого сдвига сплошной среды (рис. 4).

Как следует из работы Миндлина [5], отношение приращения силы, к вызванному этим приращением перемещению можно представить в виде:

$$
\frac{\Delta T}{\Delta \delta_{T}}=\frac{\left[6\left(1-v_{s}^{2}\right)^{2} E_{s}^{2} r_{s} T\right]^{1 / 3}}{\left(2-v_{s}\right)\left(1+v_{s}\right)}
$$

Выражая модуль упругости через модуль сдвига и коэффициент Пуассона, уравнение (10) можно представить в виде:

$$
\frac{\Delta T}{\Delta \delta_{T}}=\frac{4 \mu_{s} b}{2-v_{s}} \text {. }
$$


Опуская промежуточные выкладки, получим осреднённый модуль сдвига гранулированной среды:

$$
\tilde{\mu}_{s}=\frac{\tilde{p}_{x y}}{\tilde{\varepsilon}_{x y}}=\frac{\left[6\left(1-v_{s}^{2}\right)^{2} E_{s}^{2} r_{s} T\right]^{1 / 3}}{4 r_{s}\left(2-v_{s}\right)\left(1+v_{s}\right)}=\frac{\left[3\left(1-v_{s}^{2}\right)^{2} E_{s}^{2} \widetilde{p}\right]^{1 / 3}}{2\left(2-v_{s}\right)\left(1+v_{s}\right)} \text {. }
$$

Используя усреднённую плотность среды и полученный усреднённый модуль сдвига, найдём скорость поперечных волн в гранулированной среде:

$$
\begin{aligned}
& \beta_{s}=\sqrt{\frac{\tilde{\mu}_{s}}{\tilde{\rho}}}=\left[3\left(1-v_{s}^{2}\right)^{2} E_{s}^{2} \tilde{p}\right]^{1 / 6} \cdot\left[\frac{1}{2\left(2-v_{s}\right)\left(1+v_{s}\right)}\right]^{1 / 2} \cdot\left[\frac{6}{\pi \rho_{s}}\right]^{1 / 2}= \\
& =\left[3\left(1-v_{s}^{2}\right)^{2} E_{s}^{2} \tilde{p}\right]^{1 / 6} \cdot\left[\frac{3}{\pi \rho_{s}\left(2-v_{s}\right)\left(1+v_{s}\right)}\right]^{1 / 2} .
\end{aligned}
$$

Введя обозначения:

$$
\text { для волн сжатия } \quad A_{p}=\left[\frac{3^{4} E_{s}^{2}}{\pi^{3} \rho_{s}^{3}\left(1-v_{s}^{2}\right)^{2}}\right]^{1 / 6}
$$

для волн сдвига $A_{s}=\left[3\left(1-v_{s}^{2}\right)^{2} E_{s}^{2}\right]^{/ 6} \cdot\left[\frac{3}{\pi \rho_{s}\left(2-v_{s}\right)\left(1+v_{s}\right)}\right]^{1 / 2}$

Тогда можно записать следующие выражения определения скоростей волн сжатия и сдвига в зависимости от вертикального давления:

$$
c_{p}=A_{p} \sqrt[6]{\widetilde{p}} \text { и } c_{s}=A_{s} \sqrt[6]{\widetilde{p}} .
$$

Если предположить, что предварительное нагружение обусловлено собственным весом вышележащих слоёв грунта и весом сооружения на поверхности, тогда величина среднего давления будет определяться выражением: $\tilde{p}=\tilde{\rho} g H+P(H)$, где $g$ - ускорение свободного падения, $H$ - расстояние от
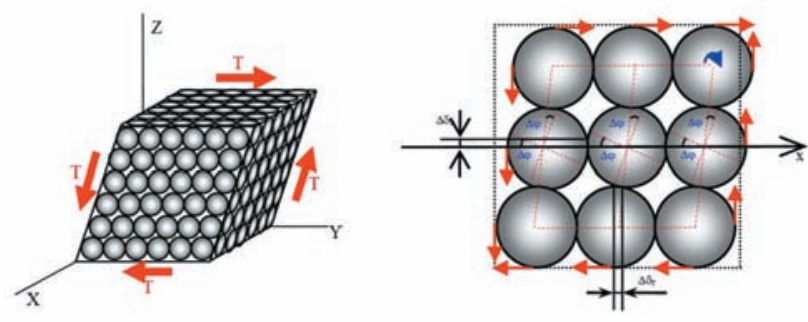

Рис. 4 Гранулированная среда, подверженная деформации простого сдвига

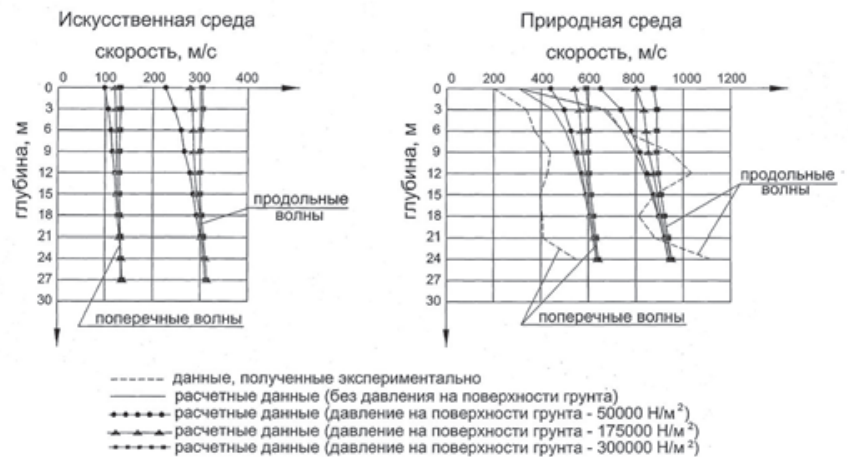

Puc. 5. Скорости распространения волн напряжений в зависимости от глубины в гранулированных средах поверхности грунта, $P(\mathrm{H})$ - давление от сооружения на глубине $H$.

Из формул (16) следует, что скорости распространения продольных и поперечных волн пропорциональны корню шестой степени от давления.

Для оценки реалистичности моделей сплошных (гранулированных) сред выполнено сравнение теоретически полученных скоростей распространения продольных и поперечных волн со скоростями распространения волн, измеренных в полевых или лабораторных условиях.

На рисунке 5 представлено сравнение значений скоростей распространения волн напряжений природной и искусственной сред с учётом и без учёта давления на свободной поверхности, полученных теоретическим путём, с результатами измерений скоростей распространения волн между скважинами в неконсолидированных песках [7].

Для сравнения скоростей распространения волн напряжений, полученных теоретических путём, с экспериментальными данными использовались следующие характеристики материалов:

$\widetilde{\rho}=\frac{\pi}{3 \sqrt{2}} \rho_{s}-$ усреднённая плотность гранулированной среды;

$\tilde{p}=\tilde{\rho} g H+P(H)-$ усреднённое давление в грунте от собственного веса грунта и сооружения;

$\rho_{\text {s1 }}=2650$ кг $/ \mathrm{m}^{3}$ - плотность песчинок кварцевого песка;

$\rho_{\mathrm{s} 2}=1800 \mathrm{\kappa г} / \mathrm{m}^{3}$ - плотность гранул из полимерного материала;

$E_{\mathrm{s} 1}=1011 \mathrm{H} / \mathrm{m}^{2}$ - модуль упругости кварца;

$E_{\mathrm{s} 2}=20,8 \cdot 10^{8} \mathrm{H} / \mathrm{M}^{2}-$ модуль упругости гранул из полимерного материала;

$v_{\mathrm{s} 1}=0,15$ - коэффициент Пуассона для песка;

$v_{\mathrm{s} 2}=0,4-$ коэффициент Пуассона для гранул из полимерного материала.

Анализ теоретических и экспериментальных результатов показывает хорошее совпадение для продольных волн. Замеренные скорости распространения поперечных волн значительно меньше теоретических значений.

Скорости распространения продольных и поперечных волн, определённые с использованием выражений (18), получаются следующие:

$\alpha_{p 1}=107 \sqrt[6]{\widetilde{p}}$ и $\beta_{s 1}=72 \sqrt[6]{\widetilde{p}}$ (для кварцевого неконсолидированного песка);

$\alpha_{p 2}=37 \sqrt[6]{\widetilde{p}}$ и $\beta_{s 2}=16 \sqrt[6]{\widetilde{p}}$ (для гранул из полимерного материала с песком).

В заключение отметим, что модели сред, состоящие из одинаковых сфер, как отмечается многими исследователями, достаточно хорошо описывают поведение неконсолидированных сред при распространении в них низкочастотных упругих волн.

Скорости распространения волн напряжений в искусственных средах, состоящих из смеси полиэтиленовых или каучуковых гранул и песка, значительно меньше скоростей 
распространения волн в природной (песчаной) среде. Из этого следует, что слои из такого материала можно использовать как для уменьшения сейсмических воздействий на сооружения, так и для защиты от техногенных вибраций.

\section{5. Виброизолирующие и сейсмоизолирующего слои}

\section{1. Зарубежный опыт}

Почти двадцать лет назад различными исследователями были предложены методы защиты сооружений от землетрясений путём использования нежёстких упругих слоёв, имеющих малое сопротивление на сдвиг.

Например, Ким (Kim) и Конагай (Konagai) (2001) [8] для уменьшения деформаций тоннелей при землетрясениях предложили наносить на обделки мягкий и тонкий синтетическим слой. Было также предложено размещать слои из синтетического материала под фундаментами сооружений или между слоями грунта для рассеивания сейсмической энергии при сдвиге (Йегиан (Yegian) и Кадакал (Kadakal), 2004 и Йегиан (Yegian) и Катан (Catan), 2004) [9; 10]. Киржнер и соавторы (2006) предложили заменить грунты, окружающие тоннель, более мягкими материалами для поглощения шума и вибрации [11]. Было предложено размещать каучуково-грунтовые смеси вокруг фундаментов строительных конструкций и подземных туннелей для поглощения сейсмической энергии и выполнения функции, аналогичной функции смягчающего мата [Цанг (Tsang), 2008; Цанг и другие, 2009] [12; 13]. Хазарика и соавторы (2008) предложили использовать измельчённые шины для защиты подпорных стен, предотвращающих разрушение берегов при землетрясении [14]. Вышеупомянутым методам сейсмоизоляции в зарубежной технической литературе присвоили специальный термин «геотехническая сейсмоизоляция», в отличие от обычно используемого термина «сейсмоизоляция» (Цанг, 2009) [13].

\section{2. Приложение метода к оценке влияния сейсмоизолирую-}

щего слоя на волны, распространяющиеся в слоистых средах

Рассмотрим распространение упругих волн природного или техногенного происхождения в слоистой упругой среде. Один из слоёв (не обязательно верхний) может быть выполнен из гранулированного материала.

Ввиду того, что время прихода продольных и поперечных волн отличается, колебания при воздействии продольных и поперечных волн рассматриваются независимо.

\section{Продольные волны}

Представим дифференциальное уравнение гармонических колебаний слоя грунта

$$
\rho \omega^{2} w+(\lambda+2 \mu) \frac{\partial^{2} w}{\partial z^{2}}=0
$$

расположенного на глубине $\left(h_{1}, h_{2}\right)$, при распространении продольных волн в обобщённых финитных функциях:

$$
\frac{\omega^{2}}{\alpha^{2}} u+\frac{\partial^{2} u}{\alpha^{2}}=\left[\frac{1}{\lambda+2 \mu} \sigma\left(h_{1}\right) \delta\left(z-h_{1}\right)-\frac{1}{\lambda+2 \mu} \sigma\left(h_{2}\right) \delta\left(z-h_{2}\right)+u\left(h_{1}\right) \dot{\delta}^{\prime}\left(z-h_{1}\right)-u\left(h_{2}\right) \dot{\delta}^{\prime}\left(z-h_{2}\right)\right],
$$

где $\omega$ - круговая частота гармонических колебаний;

$\rho, \lambda=\lambda_{0}(1+2 \xi i), \mu=\mu_{0}(1+2 \xi i)-$ плотность и коэффициенты Лямэ грунта с учётом демпфирования, равном $\xi$;

$\delta\left(z-h_{1}\right), \delta^{\prime}\left(z-h_{1}\right)-$ функция Дирака и её производная.

Применив преобразование Фурье к обеим частям уравнения (18), найдём изображение Фурье функции перемещений:

$$
\tilde{u}(v)=\frac{-i v u\left(h_{1}\right) e^{i v h_{1}}+\frac{\sigma\left(h_{1}\right)}{\lambda+2 \mu} e^{i v h_{1}}+i v u\left(h_{2}\right) e^{i v h_{2}}-\frac{\sigma\left(h_{2}\right)}{\lambda+2 \mu} e^{i v h_{2}}}{v^{2}-\frac{\omega^{2}}{\alpha^{2}}} .
$$

В соответствии с теоремой Винера-Пэли-Шварца, числитель выражения (19) должен делиться без остатка на знаменатель. Из этого следует, что он должен быть равен нулю при значениях, равных корням знаменателя. Используя это условие, получим для каждого слоя по два уравнения:

$$
\begin{aligned}
& -i v u\left(h_{1}\right) e^{i v h_{1}}+\frac{\sigma\left(h_{1}\right)}{(\lambda+2 \mu)} e^{i v h_{1}}+i v u\left(h_{2}\right) e^{i v h_{2}}-\frac{\sigma\left(h_{2}\right)}{(\lambda+2 \mu)} e^{i v h_{2}}=0 \\
& \text { при } v= \pm \eta \frac{\omega}{\alpha_{0}} .
\end{aligned}
$$

Для грунта, состоящего из $n$ слоёв, расположенных на жёсткой коренной породе, можно получить систему из $2 n$ уравнений, учитывая равенство напряжений и перемещений на границах слоёв. Кроме того, можно задать граничные условия поверхности верхнего слоя $\sigma_{0}=0$ и граничные условия на границе с коренной породой, соответствующие параметрам падающей продольной волны. Таким образом, можно получить систему $2 n$ уравнений с $2 n$ неизвестными: $M[\bar{X}]=[\bar{D}]$, где неизвестные $\bar{X}$ - перемещения и напряжения на границах слоёв; $\bar{D}$ - заданные перемещения нижней границы.

\section{Поперечные волны}

Используя в качестве исходного уравнение распространения поперечных волн и изложенную выше методику, можно получить зависимости между функциями напряжений и перемещений на границах слоёв при распространении поперечных волн:

$$
\begin{gathered}
-i v u_{\mathrm{r}}\left(h_{1}\right) e^{i v h_{1}}+\frac{\tau\left(h_{1}\right)}{\mu} e^{i v h_{1}}+i v u_{\mathrm{r}}\left(h_{2}\right) e^{i v h_{2}}-\frac{\tau\left(h_{2}\right)}{\mu} e^{i v h_{2}}=0 \\
\text { при } v= \pm \eta \frac{\omega}{\beta_{0}} ; \text { где } \eta=1-i \xi, \beta_{0}=\sqrt{\frac{\mu_{0}}{\rho}} .
\end{gathered}
$$

Изложенную методику можно использовать для оценки сейсмоизолирующих и виброизолирующих свойств гранулированного слоя грунта. Для этой цели необходимо просчитать уровни вибраций поверхности или уровни вибраций грунта на отметке расположения фундамента сооружения при наличии виброизолирующего слоя и при отсутствии этого слоя.

Отметим следующий важный факт: в некоторых полосах частот возможно усиление колебаний грунта вследствие резонанса. Для исключения отрицательного резонансного эффекта необходимо проводить расчёт и экспериментальную проверку.

\section{3. Пример сейсмоизоляции сооружения}

Метод сейсмоизоляции, основанный на свойствах изображений Фурье финитных функций, был доложен на международной конференции в Тайпее [3]. В качестве примера был представлен расчёт сейсмоизоляции атомной станции. За ис- 
ходное воздействие были приняты акселерограмма и спектр Фурье землетрясения Цзицзи 1999 года. Рассматривались два варианта: сооружение без сейсмоизоляции и сооружение с сейсмоизолирующим слоем. В качестве сейсмоизоляции использовался слой из специального синтетического материала с заданными характеристиками.

Анализ результатов расчёта показал, что большинство параметров сейсмического воздействия на фундамент с сейсмоизоляцией снижаются в несколько раз по сравнению с параметрами сейсмического воздействия на фундамент без изоляции. Несмотря на то, что в данном расчёте использовался специальный синтетический материал, полученный результат представляет интерес. Так как из бытовых отходов и использованных автомобильных шин можно создать материал, обладающий необходимыми для использования в сейсмоизоляции характеристиками.

Рассматриваемые в статье материалы могут применяться для виброизоляции зданий и сооружений от техногенных вибраций, пример виброизоляции приведен на рисунке 7.

\section{Заключение}

1. Представлен метод расчёта сейсмоизоляции и виброизоляции, основанный на свойствах изображений Фурье финитных функций.

2. Предложенный метод построения конечных элементов обладает следующими преимуществами по сравнению с используемыми конечными элементами:

- нет необходимости нелогичного моделирования сплошных сред сосредоточенными массами;
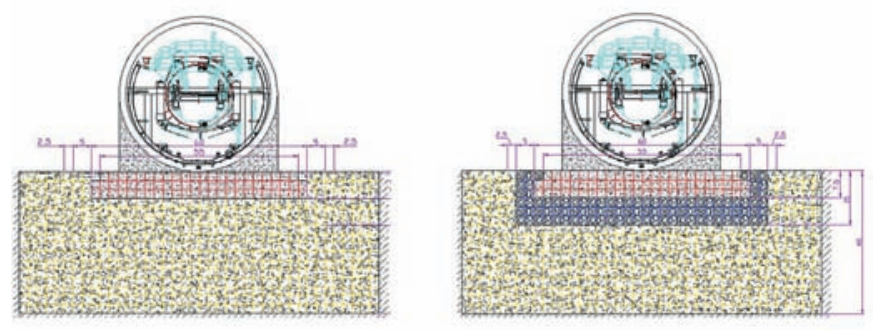

Pис. 6. Расчётные схемы на сейсмическое воздействие без сейсмоизоляции и сейсмоизолирующим слоем

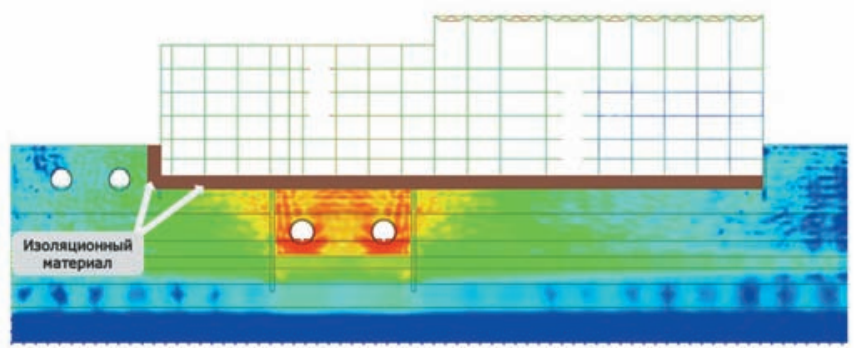

Рис. 7. Виброизоляция жилого здания от вибраций, генерируемых поездами метрополитена
- неизвестными параметрами алгебраических систем уравнений являются напряжения и перемещения на границах элементов;

- представляется возможность создавать полубесконечные элементы для описания прозрачных границ;

- имеется возможность создания трёхмерных элементов для решения пространственных задач.

3. Метод позволяет решать задачи взаимодействия сооружений с основанием во временной и частотной областях.

4. Для решения задач может использоваться алгоритм быстрого преобразования Фурье, позволяющий быстро обрабатывать большие массивы чисел.

5. Представлен метод оценки динамических свойств гранулированного материала из полимерных бытовых отходов и использованных автомобильных шин.

6. В будущем полимерные бытовые материалы могут стать неиссякаемым источником для получения материала, обладающего сейсмоизолирующими и виброизолирующими свойствами.

7. Разработка способов использования полимерных бытовых отходов для сейсмоизоляции и виброизоляции позволит одновременно решать две стоящие перед населением всего мира проблемы: защищать от загрязнений окружающую среду и обеспечивать сейсмостойкость сооружений.

\section{Лuтература}

1. Курбацкий, Е.Н. Метод решения задач строительной механики и теории упругости, основанный на свойствах изображений Фурье финитных функций : диссертация ... доктора технических наук : 05.23.17. - Б. М., 1995. - 205 с. : ил.

2. Hurgin Y.I., Yakovlev V.P. Finite Functions in Physics and Engineering. - M. : Nauka. Phismathgis, 1971, 408 pp.

3. Kurbatskiy, E.N., 2014. Seismic Abatement Method for Nuclear Power Plants and Seismic-Isolation Systems for Structural Elements // Infrastructure Systems for Nuclear Energy, First Edition. Edited by Thomas T. C. Hsu, Chiun-Lin Wu and Jui-Liang Lin - John Wiley \& Sons, Ltd. Published 2014 Ltd. P. 51-61

4. Курбацкий Е.Н. Особенности распространения волн напряжений в природных и искусственных гранулированных средах / Е.Н. Курбацкий, 0.А. Голосова // Строительная механика и расчёт сооружений. - 2011. - № 2.

5. Mindlin, R.D. Compliance of Elastic Bodies in Contact / R.D Mindlin // J. Appl. Mech., Trans. ASME. - 1949. - Vol. 71. - P. 259-268.

6. Duffy, J. Stress-Strain Relations and Vibrations of a Granular Medium / J. Duffy, R.D. Mindlin // J. Appl. Mech. 1957. - Vol. 24. - P. 585-593.

7. White J.E. Velocity Measurements in Near-surface Formations / J. E. White, R.L. Sengbush // Geophysics. - 1953. - Vol. 18. - P. 54-69.

8. Kim, D.S. Key Parameters Governing the Performance of Soft Tunnel Coating for Seismic Isolation / Kim D.S., K. Konagai 
// Earthquake Engrg. Struct. Dyn. - 2001. -Vol. 30. - №. 9. - P. 1333-1343.

9. Yegian M.K. Foundation Isolation for Seismic Protection Using a Smooth Synthetic Liner / M.K Yegian, U. Kadakal - J. Geotechnical and Geoenvironmental Engineering, ASCE. - 2004. - Vol. 130. - № 11. - P. 1121-1130.

10. Yegian, M.K. Soil Isolation for Seismic Protection Using a Smooth Synthetic Liner / M.K. Yegian, M. Catan // J. Geotechnical and Geoenvironmental Engineering, ASCE. - 2004. - Vol. 130. - № 11. - P. 1131-1139.

11. Kirzhner, F. Attenuation of Noise and Vibration Caused by Underground Trains, Using Soil Replacement / F. Kirzhner, G. Rosenhouse, Y. Zimmels // Tunnelling and Underground Space Technology. - 2006. - Vol. 21. - № 5. - P. 561-567.

12. Tsang, H.H. Geotechnical Seismic Isolation / H.H. Tsang // Earthquake Engineering: New Research/ - New York, U.S : Nova Science Publishers, Inc. - P. 55-87.

13. Underground Tunnel by Rubber-Soil Mixtures / Tsang H.H., J.Y.K. Lam, S. Yaghmaei-Sabegh and S.H. Lo // Proc. Seventh Intern. Conf. on Lifeline Earthquake Engineering, ASCETCLEE. - Oakland, California, U.S., 2009.

14. Hazarika H. Underwater Shake Table Tests on Waterfront Structures Protected with Tire Chips Cushion / H. Hazarika E., Kohama, T. Sugano // J. Geotechnical and Geoenvironmental Engineering, ASCE. - 2008. - Vol. 134. - № 12. - P. 1706-1719.

15. Geotechnical seismic isolation by scrap tire-soil mixtures / Hing-Ho Tsang Nelson T.K. Saman Yaghmaei-Sabegh, M. Neaz Sheikh, Buddhima Indraratna // Internatioal Conference. Resent Advances in Geotechnical Earthquake Engineering and Soil Mechanic. - San Diego, California, 2010.

\section{References}

1. Kurbatskii E.N. Metod resheniya zadach stroitel'noi mekhaniki i teorii uprugosti, osnovannyi na svoistvakh izobrazhenii Fur'e finitnykh funktsii [Method for solving problems of structural mechanics and the theory of elasticity based on the properties of the finite functions Fourier transforms], dissertatsiya ... doktora tekhnicheskikh nauk : 05.23.17. - B. m., 1995. - 205 s. : il.

2. Hurgin Y.I., Yakovlev V.P. Finite Functions in Physics and Engineering. Moscow, Nauka Phismathgis Publ., 1971, 408 p.

3. Kurbatskiy E.N., 2014. Seismic Abatement Method for Nuclear Power Plants and Seismic-Isolation Systems for Structural Elements. Infrastructure Systems for Nuclear Energy, First Edition. Thomas T.C. Hsu, Chiun-Lin Wu and Jui-Liang Lin (eds.). John Wiley \& Sons, Ltd. Published, 2014, pp. 51-61.
4. Kurbatskii E.N., Golosova 0.A. Osobennosti rasprostraneniya voln napryazhenii v prirodnykh $i$ iskusstvennykh granulirovannykh sredakh [Features of the propagation of stress waves in natural and artificial granular media]. Stroitel'naya mekhanika $i$ raschet sooruzhenii [Structural mechanics and calculation of structures], 2011, no. 2.

5. Mindlin R. D. Compliance of Elastic Bodies in Contact. J. Appl. Mech., Trans. ASME, 1949, Vol. 71, pp. 259-268.

6. Duffy J. R.D. Mindlin. Stress-Strain Relations and Vibrations of a Granular Medium J. Appl. Mech, 1957, Vol. 24, pp. 585-593.

7. WhiteJ.E.,Sengbush R.L., WhiteJ.E. Velocity Measurements in Near-surface Formations. Geophysics, 1953, Vol. 18, pp. 54-69.

8. Kim D.S., Konagai K. Key Parameters Governing the Performance of Soft Tunnel Coating for Seismic Isolation. Earthquake Engrg. Struct. Dyn., 2001, Vol. 30, no. 9, pp. 1333-1343.

9. Yegian M.K., Kadakal U. Foundation Isolation for Seismic Protection Using a Smooth Synthetic Liner. J. Geotechnical and Geoenvironmental Engineering, ASCE, 2004, Vol. 130, no. 11, pp. 1121-1130.

10. Yegian M.K., Catan M. Soil Isolation for Seismic Protection Using a Smooth Synthetic Liner. J. Geotechnical and Geoenvironmental Engineering, ASCE, 2004, Vol. 130, no. 11, pp. 1131-1139.

11. Kirzhner F., Rosenhouse G., Zimmels Y. Attenuation of Noise and Vibration Caused by Underground Trains, Using Soil Replacement. Tunnelling and Underground Space Technology, 2006, Vol. 21, no. 5, pp. 561-567.

12. Tsang H.H. Geotechnical Seismic Isolation. Earthquake Engineering: New Research, New York, U.S, Nova Science Publishers, Inc., pp. 55-87.

13. Tsang H.H., Lam J.Y.K., Yaghmaei-Sabegh S., Lo S.H. Underground Tunnel by Rubber-Soil Mixtures. Proc. Seventh Intern. Conf. on Lifeline Earthquake Engineering, ASCE-TCLEE. Oakland, California, U.S. Publ., 2009.

14. Hazarika H. Kohama E., Sugano T. Underwater Shake Table Tests on Waterfront Structures Protected with Tire Chips Cushion. J. Geotechnical and Geoenvironmental Engineering, ASCE, 2008, Vol. 134, no. 12, ppP. 1706-1719.

15. Hing-Ho Tsang, T.K. Nelson, Saman Yaghmaei-Sabegh, Neaz Sheikh M., Buddhima Indraratna. Geotechnical seismic isolation by scrap tire-soil mixtures. Internatioal Conference. Resent Advances in Geotechnical Earthquake Engineering and Soil Mechanic. San Diego, California, 2010. San Diego, California, 2010.

Курбацкий Евгений Николаевич (Москва) Доктор технических наук, академик РАТ. Профессор кафедры «Мосты и тоннели» ФГАОУ ВО «Российский университет транспорта» (МИИТ). (127994, Москва. ул. Образцова, д. 9, стр. 9. МИИт). Эл.почта: dynamic.@gmail.com. 
Титов Евгений Юрьевич (Москва). Кандидат технических наук. Доцент кафедры «Мосты и тоннели» ФГАОУ ВО «Российский университет транспорта» (МИИТ) (127994, Москва. ул. Образцова, д. 9, стр. 9. МИИТ), заместитель председателя - учёный секретарь Объединённого ученого совета ОАО «РЖД» (129626, Москва, 3-я Мытищинская ул., д. 10. ВНИИЖТ). Эл.почта: etitov80@gmail.com.

Голосова Ольга Андреевна (Москва). Старший преподаватель кафедры «Автомобильные дороги, аэродромы, основания и фундаменты» ФГАОУ В0 «Российский университет транспорта» (МИИТ). (127994, Москва. ул. Образцова, д. 9 стр. 9. МИИТ). Эл.почта: olga-golosova@yandex.ru.

Харитонов Сергей Сергеевич (Москва). Аспирант кафедры «Мосты и тоннели» ФГАОУ ВО «Российский университет транспорта» (МИИТ) (127994, Москва. ул. Образцова, д. 9, стр. 9. МИИТ). E-mail: mr.kharitonov.94@mail.ru.

Kurbatskiy Evgeny Nikolaevich (Moscow). Doctor of Technical Sciences, Academician of the Russian Academy of Transport. Professors of the Department of Bridges and Tunnels of FGAOU V0 “Russian Academy of Transport" (MIIT) (Obraztsova st. building 9. Moscow, 127994. MIIT). E-mail:dynamic.@gmail.com.

Titov Evgeny Yuryevich (Moscow). Candidate of Technical Sciences. Associate Professor at the Department of Bridges and Tunnels of FGAOU V0 “Russian Academy of Transport” (MIIT) (Obraztsova st. building 9. Moscow, 127994. MIIT). Deputy Chairman - Scientific Secretary of the Joint Scientific Council of Russian Railways (Russia, Moscow, 129626, 3rd Mytischinskaya Street, 10. VNIIZhT).E-mail: etitov80@gmail.com.

Golosova Olga Andreevna (Moscow). Senior lecturer of the department of Motor roads, airfields, soles and foundations of FGAOU V0 “Russian Academy of Transport” (MIIT) (Obraztsova st. building 9. Moscow, 127994. MIIT). E-mail: olga-golosova@yandex.ru.

Kharitonov Sergey Sergeevich (Moscow). Postgraduate student of the Department of Bridges and Tunnels of FGA0U V0 “Russian Academy of Transport" (MIIT) (Obraztsova st. building 9. Moscow, 127994. MIIT). E-mail: mr.kharitonov.94@mail.ru. 